\title{
Dynamics of Lotka-Volterra diffusion-advection competition system with heterogeneity vs homogeneity
}

\author{
Benlong Xu*, Hongyan Jiang \\ Department of Mathematics, Shanghai Normal University, Shanghai, 200234, P. R. China.
}

Communicated by A. Atangana

\begin{abstract}
This paper mainly studies the dynamics of a Lotka-Volterra reaction-diffusion-advection model for two competing species which disperse by both random diffusion and advection along environmental gradient. In this model, the species are assumed to be identical except spatial variation: one lives in the heterogeneity environment, the other lives in the homogeneity environment. The main results of this paper are two fold: (i) The species living in homogeneous environment can never wipe out their competitor; (ii) Explore the condition on the diffusion and advection rates for exclusion and coexistence. It is proved that for fixed dispersal rates, when the strength of the advection is sufficiently strong, the two competitive species coexist. This is a remarkable different result with that obtained by $\mathrm{He}$ and $\mathrm{Ni}$ recently for corresponding systems without advection [X. He, W.-M. Ni, J. Differential Equations, 254 (2013), 528-546]. (C)2017 All rights reserved.
\end{abstract}

Keywords: Advection, linear stability, global asymptotic stability, spatial heterogeneity, coexistence. 2010 MSC: 35B40, 35K57, 92D25.

\section{Introduction}

The question of how the interactions between spatial heterogeneity and the organism's dispersing affect the evolution of the population has fascinated ecologists and evolutionary biologists for many decades. For reaction-diffusion model, Hastings [13] and Dockery et al. [10] showed that, for two competing species with different (random) dispersal rate but otherwise identical in a heterogeneous environments, the slower diffuser always wins. To be more precise, consider the following Lotka-Volterra competition-diffusion system ([10])

$$
\begin{cases}\mathrm{U}_{\mathrm{t}}=\mathrm{d}_{1} \Delta \mathrm{U}+\mathrm{U}(\mathrm{m}(\mathrm{x})-\mathrm{U}-\mathrm{V}), & \text { in } \Omega \times \mathbb{R}^{+}, \\ \mathrm{V}_{\mathrm{t}}=\mathrm{d}_{2} \Delta \mathrm{V}+\mathrm{V}(\mathrm{m}(\mathrm{x})-\mathrm{U}-\mathrm{V}), & \text { in } \Omega \times \mathbb{R}^{+}, \\ \partial_{v} \mathrm{U}=\partial_{v} V=0, & \text { on } \partial \Omega \times \mathbb{R}^{+}, \\ \mathrm{U}(\mathrm{x}, 0)=\mathrm{U}_{0}(\mathrm{x}), \mathrm{V}(\mathrm{x}, 0)=\mathrm{V}_{0}(\mathrm{x}), & \text { in } \Omega,\end{cases}
$$

where the migration rates $d_{1}, d_{2}$ are two positive constants, $U(x, t), V(x, t)$ represent the densities of two species at location $x$ and time $t$, and $m(x)$ represents the intrinsic growth rates of species, which also

\footnotetext{
*Corresponding author

Email addresses: bxu@shnu.edu.cn (Benlong Xu), 961089322@qq.com (Hongyan Jiang)
} 
reflects the environmental richness of the resources at location $x$. The habitat $\Omega$ is a bounded region in $\mathbb{R}^{N}$ with smooth boundary $\partial \Omega, \partial_{v}=v \cdot \nabla$, where $v$ denotes the unit normal vector on $\partial \Omega$, and the no-flux boundary condition means no individuals cross the boundary. For simplicity, we will assume throughout this paper that the initial data $\mathrm{U}_{0}$ and $\mathrm{V}_{0}$ are nonnegative and nontrivial, i.e., not identically zero.

Let $r(x) \in C^{\alpha}(\bar{\Omega})(\alpha \in(0,1))$ with $\int_{\Omega} r(x) d x \geqslant 0$ and $r(x) \not \equiv 0$. It is well-known that the problem

$$
\mathrm{d} \Delta \theta+\theta(\mathrm{r}(\mathrm{x})-\theta)=0 \text { in } \Omega, \quad \partial_{\gamma} \theta=0 \text { on } \partial \Omega,
$$

has a unique positive solution (see, e.g., [3]), which is denoted by $\theta_{d, r}$.

Then the following remarkable result is established by Hastings [13] and Dockery et al. [10].

Theorem 1.1. Suppose that $0<\mathrm{m}(\mathrm{x}) \not \equiv$ const on $\bar{\Omega}$ and $\mathrm{m}(\mathrm{x}) \in \mathrm{C}^{\alpha}(\bar{\Omega})(\alpha \in(0,1))$. Then the semitrivial steady state $\left(\theta_{\mathrm{d}_{1}, \mathrm{~m}}, 0\right)$ of $(1.1)$ is globally asymptotically stable when $\mathrm{d}_{1}<\mathrm{d}_{2}$; i.e., every solution $(\mathrm{U}, \mathrm{V})$ of $(1.1)$ converges to $\left(\theta_{\mathrm{d}_{1}, \mathrm{~m}}, 0\right)$ as $\mathrm{t} \rightarrow \infty$ regardless of initial values $\left(\mathrm{U}_{0}, \mathrm{~V}_{0}\right)$.

An intuitive explanation for this surprising result is that slow diffusion helps species to better track favorable regions whereas fast diffusion will move individuals away from such ideal regions and in so doing lose certain competitive advantages.

Recently, by allowing the species $\mathrm{U}$ and $\mathrm{V}$ to have different intrinsic growth rates or to different distributions of resources, in a series of works, He and $\mathrm{Ni}$ [14-17] studied the following Lotka-Volterra model of competition-diffusion system:

$$
\begin{cases}U_{t}=d_{1} \Delta U+U\left(m_{1}(x)-U-c V\right), & \text { in } \Omega \times \mathbb{R}^{+} \\ V_{t}=d_{2} \Delta V+V\left(m_{2}(x)-b U-V\right), & \text { in } \Omega \times \mathbb{R}^{+} \\ \partial_{v} U=\partial_{v} V=0, & \text { on } \partial \Omega \times \mathbb{R}^{+} \\ U(x, 0)=U_{0}(x), \quad V(x, 0)=V_{0}(x), & \text { in } \Omega\end{cases}
$$

where $m_{1}(x)$ and $m_{2}(x)$ represent the carrying capacities or intrinsic growth rates, which reflect the environmental influence on the species $\mathrm{U}$ and $\mathrm{V}$, respectively. The positive constants $\mathrm{b}$ and $\mathrm{c}$ are interspecific competition coefficients, while both intra-specific competition coefficients are normalized to 1 .

On the other hand, reaction-diffusion-advection equations nowadays seem more and more popular in spatial population dynamics. Belgacem and Cosner in [2] firstly proposed the single species model in the situation where individuals are very smart so that they can sense and follow gradients in resource distribution, and then Cantrell et al. [4] analyzed the corresponding two-species model. This topic has received considerable research attention; see, e.g., [3-9, 12, 22-24, 28] and the references therein, for some latest advances, see [1].

Motivated by the previous works, we introduce the following coupled reaction-diffusion-advection system

$$
\begin{cases}\mathrm{U}_{\mathrm{t}}=\nabla \cdot\left(\mathrm{d}_{1} \nabla \mathrm{U}-\alpha \mathrm{U} \nabla \mathrm{m}_{1}\right)+\mathrm{U}\left(\mathrm{m}_{1}(\mathrm{x})-\mathrm{U}-\mathrm{c} \mathrm{V}\right), & \text { in } \Omega \times \mathbb{R}^{+}, \\ \mathrm{V}_{\mathrm{t}}=\nabla \cdot\left(\mathrm{d}_{2} \nabla \mathrm{V}-\beta \mathrm{V} \nabla \mathrm{m}_{2}\right)+\mathrm{V}\left(\mathrm{m}_{2}(\mathrm{x})-\mathrm{b} \mathrm{U}-\mathrm{V}\right), & \text { in } \Omega \times \mathbb{R}^{+}, \\ \left(\mathrm{d}_{1} \nabla \mathrm{U}-\alpha \mathrm{U} \nabla \mathrm{m}_{1}\right) \cdot v=\left(\mathrm{d}_{2} \nabla \mathrm{V}-\beta \mathrm{V} \nabla \mathrm{m}_{2}\right) \cdot v=0, & \text { on } \partial \Omega \mathbb{R}^{+}, \\ \mathrm{U}(\mathrm{x}, 0)=\mathrm{U}_{0}(\mathrm{x}), \quad \mathrm{V}(\mathrm{x}, 0)=\mathrm{V}_{0}(\mathrm{x}) & \text { in } \Omega,\end{cases}
$$

where $\alpha, \beta$ which are positive constants measure the speed of movement upward along the gradient of resources $m_{1}(x)$ and $m_{2}(x)$, respectively. Our main concern in this paper is to pursue the dynamics system (1.3), especially the affect of advection rate $\alpha, \beta$ on the dynamics of this system.

When $\alpha=\beta=0$, system (1.3) becomes the system (1.2), which is studied by He and $\mathrm{Ni}$ [14-17] recently. By detailed computation and analysis, He and $\mathrm{Ni}$ [14-17] obtained some dramatic picture of global dynamics of (1.2) based on diffusion rates $d_{1}$ and $d_{2}$. Especially, for the case of heterogeneity vs. homogeneity with equal amount of total resources and $b=1, c=1$, He and Ni [14, 17] obtained thoroughly complete global dynamics of (1.2). More precisely, in [14, 17], He and Ni proposed the 
following system

$$
\begin{cases}\mathrm{U}_{\mathrm{t}}=\mathrm{d}_{1} \Delta \mathrm{U}+\mathrm{U}(\mathrm{m}(\mathrm{x})-\mathrm{U}-\mathrm{V}), & \text { in } \Omega \times \mathbb{R}^{+}, \\ \mathrm{V}_{\mathrm{t}}=\mathrm{d}_{2} \Delta V+\mathrm{V}(\overline{\mathrm{m}}-\mathrm{U}-\mathrm{V}), & \text { in } \Omega \times \mathbb{R}^{+}, \\ \partial_{v} \mathrm{U}=\partial_{v} V=0, & \text { on } \partial \Omega \times \mathbb{R}^{+}, \\ \mathrm{U}(x, 0)=\mathrm{U}_{0}(x), \mathrm{V}(\mathrm{x}, 0)=\mathrm{V}_{0}(\mathrm{x}), & \text { in } \Omega,\end{cases}
$$

where $m(x)$ is non-constant in $\Omega$ and $\bar{m}$ is the average density of the resources $m(x)$, i.e.,

$$
\bar{m}=\frac{1}{|\Omega|} \int_{\Omega} m(x) d x .
$$

The following notations of subsets of the first quadrant of the $d_{1} d_{2}$-plane for (1.4) is also used in $[14,17]$ :

$$
\begin{aligned}
& \Sigma_{\mathrm{u}}:=\left\{\left(\mathrm{d}_{1}, \mathrm{~d}_{2}\right) \in \mathcal{Q} \mid\left(\theta_{\mathrm{d}_{1}, \mathrm{~m}}, 0\right) \text { is linearly stable }\right\} \\
& \Sigma_{\mathrm{V}}:=\left\{\left(\mathrm{d}_{1}, \mathrm{~d}_{2}\right) \in \mathcal{Q} \mid(0, \overline{\mathrm{m}}) \text { is linearly stable }\right\} \\
& \Sigma_{-}:=\left\{\left(\mathrm{d}_{1}, \mathrm{~d}_{2}\right) \in \mathcal{Q} \mid \text { both }\left(\theta_{\mathrm{d}_{1}, \mathrm{~m}}, 0\right) \text { and }(0, \overline{\mathrm{m}}) \text { are linearly unstable }\right\},
\end{aligned}
$$

where

$$
\mathcal{Q}:=\mathbb{R}^{+} \times \mathbb{R}^{+} \quad \text { and } \quad \mathbb{R}^{+}:=(0, \infty) .
$$

For the precise definition of linear stability/instability of a steady state and their characterization, see e.g., [3]. We refer the readers to the beginning of Section 2 where the concept is defined via the sign of appropriate principal eigenvalues. He and $\mathrm{Ni}$ obtained the following remarkable characterization of global dynamics result for system (1.4).

Theorem $1.2([14,17])$. Assume that $\mathrm{m}(\mathrm{x}) \in \mathrm{C}^{\gamma}(\bar{\Omega})(\gamma \in(0,1)), \mathrm{m} \not \equiv$ const and $\mathrm{m} \geqslant 0$ on $\bar{\Omega}$, then

(i) $\Sigma_{V}=\emptyset$, namely, $(0, \bar{m})$ is always linearly unstable for all $\left(\mathrm{d}_{1}, \mathrm{~d}_{2}\right) \in \mathrm{Q}$;

(ii) $\Sigma_{\mathrm{u}} \neq \emptyset$, and $\left(\theta_{\mathrm{d}_{1}, \mathrm{~m}}, 0\right)$ is globally asymptotically stable for all $\left(\mathrm{d}_{1}, \mathrm{~d}_{2}\right) \in \overline{\Sigma_{\mathrm{u}}}$, where $\overline{\Sigma_{\mathrm{u}}}$ denotes the closure of $\Sigma_{\mathrm{u}}$ in $\mathrm{Q}$, moreover, $\Sigma_{\mathrm{u}}$ can be characterized by

$$
\Sigma_{\mathrm{u}}=\left\{\left(\mathrm{d}_{1}, \mathrm{~d}_{2}\right) \in \mathcal{Q} \mid \mathrm{d}_{2}>\widehat{\mathrm{d}}_{2}^{*}\left(\mathrm{~d}_{1}\right)\right\},
$$

where $\widehat{\mathrm{d}}_{2}^{*}\left(\mathrm{~d}_{1}\right)$ is a continuous function of $\mathrm{d}_{1}$ defined in $\mathbb{R}^{+}$with the property

$$
\widehat{\mathrm{d}}_{2}^{*}\left(\mathrm{~d}_{1}\right) \rightarrow \infty, \quad \text { as } \mathrm{d}_{1} \rightarrow 0 ; \quad \widehat{\mathrm{d}}_{2}^{*}\left(\mathrm{~d}_{1}\right) \rightarrow 0, \quad \text { as } \mathrm{d}_{1} \rightarrow \infty ;
$$

(iii) $\Sigma_{-} \neq \emptyset$, and $Q=\overline{\Sigma_{\mathrm{u}}} \cup \Sigma_{-}$, moreover, (1.4) has a unique coexistence steady state which is globally asymptotically stable for all $\left(\mathrm{d}_{1}, \mathrm{~d}_{2}\right) \in \Sigma_{-}=Q \backslash \overline{\Sigma_{\mathrm{u}}}$.

Theorem 1.2 implies that for two competitive species having identical competition abilities and the same amount of total resources, the species with spatial heterogeneous distribution are always in a superior position to their homogeneous counterpart: it is always guaranteed to survive, and it will often wipe out its competitor, so long as the diffusion point $\left(d_{1}, d_{2}\right)$ is above the critical line $d_{2}=\widehat{d}_{2}^{*}\left(d_{1}\right)$.

Following the idea of $[15,17]$, let $m_{1}(x)=m(x), m_{2}(x)=\bar{m}=\frac{1}{|\Omega|} \int_{\Omega} m(x) d x$, and $b=c=1$, then system (1.3) changes to the following coupled reaction-diffusion-advection system

$$
\begin{cases}\mathrm{U}_{\mathrm{t}}=\nabla \cdot\left(\mathrm{d}_{1} \nabla \mathrm{U}-\alpha \mathrm{U} \nabla \mathrm{m}\right)+\mathrm{U}(\mathrm{m}(\mathrm{x})-\mathrm{U}-\mathrm{V}), & \text { in } \Omega \times \mathbb{R}^{+}, \\ \mathrm{V}_{\mathrm{t}}=\mathrm{d}_{2} \Delta \mathrm{V}+\mathrm{V}(\overline{\mathrm{m}}-\mathrm{U}-\mathrm{V}), & \text { in } \Omega \times \mathbb{R}^{+}, \\ \left(\mathrm{d}_{1} \nabla \mathrm{U}-\alpha \mathrm{U} \nabla \mathrm{m}\right) \cdot \mathrm{v}=\partial_{\mathrm{v}} \mathrm{V}=0, & \text { on } \Omega \times \mathbb{R}^{+}, \\ \mathrm{U}(\mathrm{x}, 0)=\mathrm{U}_{0}(\mathrm{x}), \quad \mathrm{V}(\mathrm{x}, 0)=\mathrm{V}_{0}(\mathrm{x}), & \text { in } \Omega .\end{cases}
$$

Note that since $m_{2}(x)=\bar{m}$ is a constant, the gradient $\nabla \bar{m} \equiv 0$, and then advection rate $\beta$ has no effect to the system (1.5). As in $[15,17]$, we assume $m(x)$ satisfies the assumption $(M)$.

We assume that the initial data $U_{0}, V_{0}$ of (1.5) are non-negative and not identically zero, then by maximum principle [29], we can obtain $U>0, V>0$.

We assume that 
(M) $m(x) \in C^{2+\gamma}(\bar{\Omega})(\gamma \in(0,1)), \quad m \neq \equiv$ const and $m \geqslant 0$ on $\bar{\Omega}$.

Under assumption (M), (1.5) has two semi-trivial steady states for all $d_{1}, d_{2}>0$ and $\alpha \geqslant 0$ ([3]), denoted by $(\tilde{u}, 0),(0, \bar{m})$, respectively, where $\tilde{u}$ is the unique positive solution of

$$
\begin{cases}\nabla \cdot\left(\mathrm{d}_{1} \nabla \tilde{\mathrm{u}}-\alpha \tilde{\mathrm{u}} \nabla \mathrm{m}\right)+\tilde{\mathrm{u}}(\mathrm{m}(\mathrm{x})-\tilde{\mathrm{u}})=0, & \text { in } \Omega, \\ \left(\mathrm{d}_{1} \nabla \tilde{u}-\alpha \tilde{u} \nabla \mathrm{m}\right) \cdot v=0, & \text { on } \partial \Omega,\end{cases}
$$

and $\bar{m}$ is the unique positive solution of

$$
\begin{cases}\mathrm{d}_{2} \Delta \tilde{v}+\tilde{v}(\overline{\mathrm{m}}-\tilde{v})=0, & \text { in } \Omega, \\ \partial_{\nu} \tilde{v}=0, & \text { on } \partial \Omega\end{cases}
$$

Now we state our first result.

Theorem 1.3. Suppose that assumption (M) holds. Then $(0, \overline{\mathrm{m}})$ is unstable for every $\mathrm{d}_{1}, \mathrm{~d}_{2}$, and $\alpha \geqslant 0$.

Theorem 1.3 implies that for every $\alpha \geqslant 0$, the species $V$ can never exclude the species $U$.

Theorem 1.4. Suppose that assumption (M) holds. Then for every fixed $\mathrm{d}_{1}>0,(\tilde{\mathrm{u}}, 0)$ is globally asymptotically stable for all $\mathrm{d}_{2}$ sufficiently large and $\alpha$ sufficiently small.

Theorem 1.4 implies that for small $\alpha$ and large $d_{2}$, species $U$ always prevails.

Theorem 1.5. Suppose that assumptions (M) holds. Then for any fixed $\mathrm{d}_{1}>0$, there exists a constant $\alpha^{*}=$ $\alpha^{*}\left(\mathrm{~d}_{1}\right)>0$, such that if $\alpha>\alpha^{*},(\tilde{\mathrm{u}}, 0)$ is unstable for every $\mathrm{d}_{2}>0$.

Since the system (1.5) is a monotone system, by the theory of monotone system [20], we have the following coexistence result.

Theorem 1.6. Suppose that assumptions (M) holds. Then for fixed $\mathrm{d}_{1}>0$ and $\alpha$ sufficiently large, the problem (1.5) has at least one stable positive coexistence steady state for all $\mathrm{d}_{2}>0$.

Theorem 1.6 implies that for fixed $d_{1}>0$, species $U$ can never wipe out its competitor $V$ for all $d_{2}>0$, if species $U$ adopts sufficiently strong advection. This is a different phenomenon to that obtained in [14] as stated in the previous passages.

The rest of this paper is organized as follows. In Section 2 we collect some preliminaries that will be used in the following sections. The main results, Theorems 1.3-1.6, are proved in Section 3. Finally, some concluding remarks are included in Section 4.

\section{Preliminaries}

To study the dynamics of system (1.5), we should study the stability of semi-trivial steady states $(\tilde{u}, 0),(0, \bar{m})$. Mathematically, the stability of $(\tilde{u}, 0)$ is determined by the principal eigenvalue, denoted by $\mu_{1}\left(d_{2}, m-\tilde{u}\right)$, of the elliptic eigenvalue problem

$$
\begin{cases}\mathrm{d}_{2} \Delta \psi+(\overline{\mathrm{m}}-\widetilde{\mathrm{u}}) \psi+\mu \psi=0, & \text { in } \Omega, \\ \partial_{\nu} \psi=0, & \text { on } \partial \Omega\end{cases}
$$

Similarly, the stability of $(0, \bar{m})$ is determined by the principal eigenvalue, denoted by $\lambda_{1}\left(d_{1}, \alpha, m-\bar{m}\right)$, of the linear problem as follows:

$$
\begin{cases}\nabla \cdot\left(\mathrm{d}_{1} \nabla \psi-\alpha \psi \nabla \mathrm{m}\right)+\psi(\mathrm{m}-\overline{\mathrm{m}})+\lambda \psi=0, & \text { in } \Omega \\ \left(\mathrm{d}_{1} \nabla \psi-\alpha \psi \nabla \mathrm{m}\right) \cdot v=0, & \text { on } \partial \Omega\end{cases}
$$

More precisely, we have the following well-known criterion. 
Lemma 2.1 ([3]). ( $\tilde{u}, 0)$ is linearly stable if $\mu_{1}\left(\mathrm{~d}_{2}, \mathrm{~m}-\tilde{\mathrm{u}}\right)>0$ and is linearly unstable if $\mu_{1}\left(\mathrm{~d}_{2}, \mathrm{~m}-\tilde{\mathrm{u}}\right)<0$. Similarly, $(0, \bar{m})$ is linearly stable if $\lambda_{1}\left(\mathrm{~d}_{1}, \alpha, \mathrm{m}-\overline{\mathrm{m}}\right)>0$ and is linearly unstable if $\lambda_{1}\left(\mathrm{~d}_{1}, \alpha, \mathrm{m}-\overline{\mathrm{m}}\right)<0$.

The following result is due to [1, Lemma 4.1] some similar results can be seen in [26] for more general m.

Lemma 2.2. Assume that $\mathrm{m}(\mathrm{x}) \in \mathrm{C}^{2}(\bar{\Omega}), \mathrm{m} \not \equiv$ constant, and $\int_{\Omega} \mathrm{m}(\mathrm{x}) \mathrm{d} x \geqslant 0$ ( $\mathrm{m}$ may change sign). Then the steady state problem

$$
\begin{cases}\nabla \cdot(\mathrm{d} \nabla \tilde{\mathrm{u}}-\alpha \tilde{\mathrm{u}} \nabla \mathrm{m})+\tilde{\mathrm{u}}(\mathrm{m}-\tilde{\mathrm{u}})=0, & \text { in } \Omega, \\ (\mathrm{d} \nabla \tilde{\mathrm{u}}-\alpha \tilde{u} \nabla \mathrm{m}) \cdot v=0, & \text { on } \partial \Omega .\end{cases}
$$

has a unique positive solution ũ. Moreover, for fixed $\mathrm{d}>0$,

$$
\limsup _{\alpha \rightarrow \infty} \int_{\Omega}(\tilde{u}-m) \leqslant-\int_{\{x \in \Omega:|\nabla m| \neq 0\}} m<0 .
$$

For monotone dynamical systems, we state the standard fact that linear stability (resp., linear instability) implies asymptotic stability (resp., instability) ([30]).

Lemma $2.3([25,30])$. If a steady state $(\tilde{\mathrm{U}}, \tilde{\mathrm{V}})$ of $(1.5)$ is linearly stable (resp., linearly unstable), then it is asymptotically stable (resp., unstable).

Next, we have the following lemma derived from the theory of monotone dynamical systems.

Lemma $2.4([18,25])$. For any $\mathrm{d}_{1}, \mathrm{~d}_{2}>0$, assume every coexistence steady state of $(1.5)$, if it exists, is asymptotically stable, then one of the following alternatives holds:

(a) there exists a unique coexistence steady state of (1.5) which is globally asymptotically stable;

(b) system (1.5) has no coexistence steady state, and one of $(\tilde{u}, 0)$ or $(0, \overline{\mathrm{m}})$ is globally asymptotically stable, while the other one is unstable.

\section{Proofs of the main results}

In this section, we establish the our main results.

Proof of Theorem 1.3. Let $\lambda_{1}=\lambda_{1}\left(d_{1}, \alpha, m-\bar{m}\right)$ be the principal eigenvalue of (2.1) and $\psi>0$ be the corresponding eigenfunction, then

$$
\begin{cases}\nabla \cdot\left(\mathrm{d}_{1} \nabla \psi-\alpha \psi \nabla \mathrm{m}\right)+\psi(\mathrm{m}-\overline{\mathrm{m}})+\lambda_{1} \psi=0, & \text { in } \Omega, \\ \left(\mathrm{d}_{1} \nabla \psi-\alpha \psi \nabla \mathrm{m}\right) \cdot v=0, & \text { on } \partial \Omega,\end{cases}
$$

Set $\varphi=e^{-\left(\alpha / d_{1}\right) m} \psi$, then $\varphi$ satisfies

$$
\begin{cases}d_{1} \nabla\left(e^{\left(\alpha / d_{1}\right) m} \nabla \varphi\right)+e^{\left(\alpha / d_{1}\right) m}(m-\bar{m}) \varphi+\lambda_{1} e^{\left(\alpha / d_{1}\right) m} \varphi=0, & \text { in } \Omega, \\ \partial_{\nu} \varphi=0, & \text { on } \partial \Omega .\end{cases}
$$

Dividing (3.1) by $\varphi$, integrating in $\Omega$, we have

$$
d_{1} \int_{\Omega} \frac{e^{\left(\alpha / d_{1}\right) m}|\nabla \varphi|^{2}}{\varphi^{2}}+\int_{\Omega}(m-\bar{m}) e^{\left(\alpha / d_{1}\right) m}+\lambda_{1} \int_{\Omega} e^{\left(\alpha / d_{1}\right) m}=0 .
$$

Define

$$
\Omega^{+}=\{x \in \Omega \mid m(x) \geqslant \bar{m}\}, \quad \Omega^{-}=\{x \in \Omega \mid m(x)<\bar{m}\},
$$


then $\Omega=\Omega^{+} \cup \Omega^{-}$, and

$$
\begin{aligned}
\int_{\Omega}(m-\bar{m}) e^{\left(\alpha / d_{1}\right) m} & =\int_{\Omega^{+}}(m-\bar{m}) e^{\left(\alpha / d_{1}\right) m}+\int_{\Omega^{-}}(m-\bar{m}) e^{\left(\alpha / d_{1}\right) m} \\
& >\int_{\Omega^{+}}(m-\bar{m}) e^{\left(\alpha / d_{1}\right) \bar{m}}+\int_{\Omega^{-}}(m-\bar{m}) e^{\left(\alpha / d_{1}\right) \bar{m}} \\
& =e^{\left(\alpha / d_{1}\right) \bar{m}}\left[\int_{\Omega^{+}}(m-\bar{m})+\int_{\Omega^{-}}(m-\bar{m})\right] \\
& =e^{\left(\alpha / d_{1}\right) \bar{m}} \int_{\Omega}(m-\bar{m})=0 .
\end{aligned}
$$

It follows from (3.2) and (3.3) that $\lambda_{1}<0$, and then by Lemma $2.1,(0, \overline{\mathrm{m}})$ is always linearly unstable.

Before we prove Theorem 1.4, we propose the following boundedness lemma.

Lemma 3.1. Suppose that $\mathrm{m} \in \mathrm{C}^{2+\gamma}(\bar{\Omega})$, and $(\tilde{\mathrm{U}}, \tilde{\mathrm{V}})$ is a positive steady state solution of (1.5), then

$$
\|\tilde{U}\|_{L^{\infty}} \leqslant\|m\|_{L^{\infty}} e^{\alpha / d_{1}\|m\|_{L^{\infty}}},\|\tilde{V}\|_{L^{\infty}} \leqslant \bar{m} .
$$

Proof. $(\tilde{\mathrm{U}}, \tilde{\mathrm{V}})$ satisfies the following elliptic system

$$
\begin{cases}\nabla \cdot\left(\mathrm{d}_{1} \nabla \tilde{U}-\alpha \tilde{U} \nabla m\right)+\tilde{U}(m(x)-\tilde{U}-\tilde{V})=0, & \text { in } \Omega, \\ d_{2} \Delta \tilde{V}+\tilde{V}(\bar{m}-\tilde{U}-\tilde{V})=0, & \text { in } \Omega, \\ \left(d_{1} \nabla \tilde{U}-\alpha \tilde{U} \nabla m\right) \cdot v=\partial_{v} \tilde{V}=0, & \text { on } \partial \Omega,\end{cases}
$$

By the maximal principle and comparison theorem $([11,29])$, we have that $\|\tilde{V}\|_{L^{\infty}} \leqslant \bar{m}$, and

$$
\|\tilde{u}\|_{L^{\infty}} \leqslant\|\tilde{u}\|_{L^{\infty}},
$$

where $\tilde{u}$ is the unique positive solution of the equation

$$
\nabla \cdot\left(\mathrm{d}_{1} \nabla \tilde{u}-\alpha \tilde{u} \nabla \mathfrak{m}\right)+\tilde{u}(\mathfrak{m}(x)-\tilde{u})=0 \text { in } \Omega, \quad\left(d_{1} \nabla \tilde{u}-\alpha \tilde{u} \nabla \mathfrak{m}\right) \cdot v=0 \text { on } \partial \Omega .
$$

Let $w(x)=e^{-\alpha / d_{1} m(x)} \tilde{u}(x)$, then (3.4) becomes

$$
\mathrm{d}_{1} \nabla \cdot\left(e^{\alpha / \mathrm{d}_{1} \mathrm{~m}} \nabla w\right)+e^{\alpha / \mathrm{d}_{1} \mathrm{~m}} w(\mathrm{~m}-\tilde{\mathrm{u}})=0 \text { in } \Omega, \quad \partial_{\nu} w=0 \text { on } \partial \Omega .
$$

Let $x_{0}$ be the global maximum point of $w(x)$, we have $\tilde{\mathfrak{u}}\left(x_{0}\right) \leqslant m\left(x_{0}\right)$ and hence sup $w=w\left(x_{0}\right)=$ $e^{-\alpha / d_{1} m\left(x_{0}\right)} \tilde{u}\left(x_{0}\right) \leqslant e^{-\alpha / d_{1} m\left(x_{0}\right)} m\left(x_{0}\right)$. Hence

$$
\|\tilde{u}(x)\|_{L^{\infty}} \leqslant \sup \left[e^{\alpha / d_{1} m(x)} w(x)\right] \leqslant \sup \left[e^{\alpha / d_{1}\left(m(x)-m\left(x_{0}\right)\right)} m\left(x_{0}\right)\right] \leqslant\|m\|_{L^{\infty}} e^{\alpha / d_{1}\|m\|_{L}} .
$$

This completes the proof.

Now we are ready to prove Theorem 1.4.

Proof of Theorem 1.4. By Theorem 1.3, the semi-trivial steady state solution $(0, \bar{m})$ is linearly unstable for all $\mathrm{d}_{1}, \mathrm{~d}_{2}, \alpha>0$, then by Lemma 2.3 and Lemma 2.4 , to show that $(\tilde{\mathrm{u}}, 0)$ is globally asymptotically stable, it suffices to show that (1.5) has no co-existence steady state for all $d_{2}$ sufficiently large and $\alpha$ sufficiently small. Suppose this is not true, then there exist some $d_{1}>0$, a positive sequence $d_{2}^{(k)}$ of $d_{2}$ with $d_{2}^{(k)} \rightarrow \infty$, a positive sequence $\alpha^{(k)}$ of $\alpha$ with $\alpha^{(k)} \rightarrow 0$, such that (1.5) has a positive coexistence steady state $\left(\tilde{U}_{\alpha^{(k)}, d_{2}^{(k)}}, \tilde{V}_{\alpha^{(k)}, d_{2}^{(k)}}\right)$.

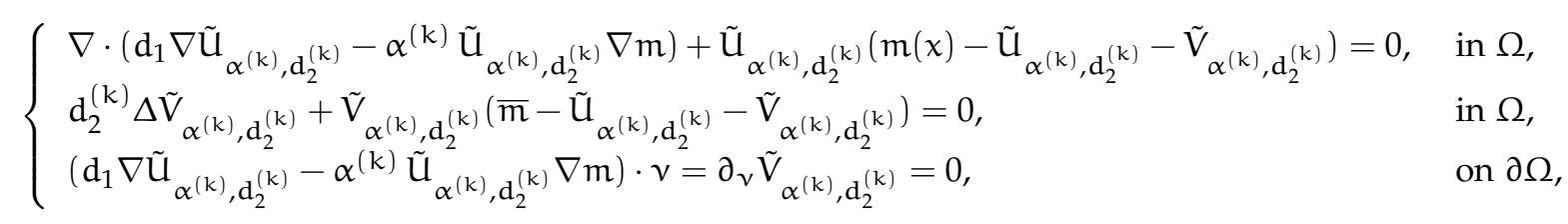


By Lemma 3.1, both $\tilde{U}_{\alpha^{(k)}, d_{2}^{(k)}}$ and $\tilde{V}_{\alpha^{(k)}, d_{2}^{(k)}}$ are uniformly bounded on $\bar{\Omega}$ independent of $d_{1}, d_{2}^{(k)}$ and $\alpha^{(k)}$. By standard $L^{p}$ regularity theory for elliptic equation, passing to their subsequences of $d_{2}^{(k)}$ and $\alpha^{(k)}$ if necessary, we may assume that

$$
\lim _{k \rightarrow \infty}\left(\tilde{U}_{\alpha^{(k)}, d_{2}^{(k)}}, \tilde{V}_{\alpha^{(k)}, d_{2}^{(k)}}\right)=\left(\tilde{U}_{\infty}, \tilde{V}_{\infty}\right) \text { in } W^{1, p}(\Omega) \times W^{1, p}(\Omega) \text { for any } p>1 .
$$

Then the Sobolev imbedding theorems and the Schauder regularity theory imply that

$$
\lim _{k \rightarrow \infty}\left(\tilde{U}_{\alpha^{(k)}, \mathrm{d}_{2}^{(k)}}, \tilde{V}_{\alpha^{(k)}, \mathrm{d}_{2}^{(k)}}\right)=\left(\tilde{\mathrm{U}}_{\infty}, \tilde{\mathrm{V}}_{\infty}\right) \text { in } \mathrm{C}^{2}(\Omega) \times \mathrm{C}^{2}(\Omega) \text {. }
$$

Dividing the second equation of (3.5) by $\mathrm{d}_{2}^{(\mathrm{k})}$ and letting $\mathrm{k} \rightarrow \infty$, we conclude that the limiting function $\tilde{V}_{\infty}$ satisfies that

$$
\Delta \tilde{V}_{\infty}=0 \quad \text { in } \Omega, \quad \partial_{\nu} \tilde{V}_{\infty}=0 \quad \text { on } \partial \Omega .
$$

Thus $\tilde{V}_{\infty} \equiv C$ for some constant $C \geqslant 0$. Setting $V_{\alpha^{(k)}, d_{2}^{(k)}}^{*}:=\frac{\tilde{V}_{\alpha^{(k)}, d_{2}^{(k)}}}{\left\|\tilde{V}_{\alpha^{(k)}, d_{2}(k)}\right\|_{L^{\infty}(\Omega)}}$, then $V_{\alpha^{(k)}, d_{2}^{(k)}}^{(k a t i s f i e s}$

$$
\Delta V_{\alpha^{(k)}, d_{2}^{(k)}}^{*}+\frac{\bar{m}-\tilde{U}_{\alpha^{(k)}, d_{2}^{(k)}}-\tilde{V}_{\alpha^{(k)}, d_{2}^{(k)}}}{d_{2}^{(k)}} V_{\alpha^{(k)}, d_{2}^{(k)}}^{*}=0 \quad \text { in } \Omega, \quad \partial_{v} V_{\alpha^{(k)}, d_{2}^{(k)}}^{*}=0 \quad \text { on } \partial \Omega
$$

By similar arguments as before, $V_{\alpha^{(k)}, d_{2}^{(k)}}^{*}$ converges to some non-negative constant $V_{\infty}^{*}$ as $k \rightarrow \infty$. Since $\left\|\mathrm{V}_{\alpha^{(\mathrm{k})}, \mathrm{d}_{2}^{(\mathrm{k})}}\right\|_{\mathrm{L}^{\infty}(\Omega)}=1, \mathrm{~V}_{\infty}^{*} \equiv 1$.

Integrating the second equation of (3.5) and then dividing by $\left\|\tilde{V}_{\alpha^{(k)}, d_{2}^{(k)}}\right\|_{L^{\infty}(\Omega)}$, we have

$$
\int_{\Omega} \mathrm{V}_{\alpha^{(k)}, \mathrm{d}_{2}^{(k)}}^{*}\left(\overline{\mathrm{m}}-\tilde{\mathrm{U}}_{\alpha^{(\mathrm{k})}, \mathrm{d}_{2}^{(k)}}-\tilde{\mathrm{V}}_{\alpha^{(\mathrm{k})}, \mathrm{d}_{2}^{(k)}}\right)=0 .
$$

Letting $k \rightarrow \infty$, we obtain that

$$
\int_{\Omega}\left(\bar{m}-\tilde{u}_{\infty}-C\right)=0
$$

Thus $\int_{\Omega}(\mathrm{m}-\mathrm{C})=\int_{\Omega}(\overline{\mathrm{m}}-\mathrm{C})=\int_{\Omega} \tilde{\mathrm{U}}_{\infty} \geqslant 0$. By letting $\mathrm{k} \rightarrow \infty$ in the first equation of (3.5), we have

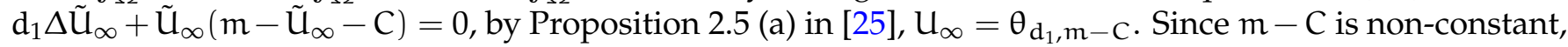
by the remarkable result obtained by Lou ([27, Theorem 1.2]), we get $\int_{\Omega} \theta_{d_{1}, m-C}>\int_{\Omega}(m-C)$ for any $\mathrm{d}_{1}>0$, which is a contradiction to (3.6).

Next, we prove Theorem 1.5.

Proof of Theorem 1.5. It suffices to show that the principal eigenvalue $\mu_{1}$ for the problem

$$
\begin{cases}\mathrm{d}_{2} \Delta \psi+(\overline{\mathrm{m}}-\tilde{\mathrm{u}}) \psi+\mu_{1} \psi=0, & \text { in } \Omega, \\ \frac{\partial \psi}{\partial \mathrm{n}}=0, & \text { on } \partial \Omega,\end{cases}
$$

is negative for $\alpha$ sufficiently large. Dividing (3.7) by $\psi$, integrating in $\Omega$, we obtain

$$
\int_{\Omega}(\tilde{u}-\bar{m})-d_{2} \int_{\Omega} \frac{|\nabla \psi|^{2}}{\psi^{2}}=\int_{\Omega} \mu_{1} .
$$

By Lemma 2.2, for any fixed $d_{1}>0$, there exists a constant $\alpha^{*}=\alpha^{*}\left(d_{1}\right)>0$, such that for any $\alpha>\alpha^{*}$, $\int_{\Omega}(\tilde{u}-\bar{m})=\int_{\Omega}(\tilde{u}-m)<0$, and hence $\mu_{1}<0$. This completes the proof.

Finally, we prove Theorem 1.6.

Proof of Theorem 1.6. Since system (1.5) is a strongly monotone system (Lemma 2.2 of [5]), it follows from Theorem 1.3, Theorem 1.5, and theory for monotone systems (see, e.g., Corollary 7.6 and Theorem 10.2 of [19]) that system (1.5) has at least one stable coexistence state. 


\section{Concluding remarks}

We study the dynamics of the Lotka-Volterra reaction-diffusion-advection model (1.3), in which the two competing species have equal total resources but different resource distribution. The two species adopt the dispersal strategy of a combination of random dispersal and biased movement upward along the resource gradient (moving to the location with better resource). Similar model defined in the whole space $\mathrm{R}^{\mathrm{n}}$ with periodic resource environment is proposed in [21].

In this paper, we mainly study the situation that one species, species $\mathrm{V}$, adopts the homogeneous distribution, and the competitor, species $U$, adopts heterogeneous distribution. In this way we obtain the system (1.5).

We are interested to understand how the resource distribution, the random dispersal rate, and the advection rate affect the dynamics of the system. We investigate this question by the sign of eigenvalue of two semi-trivial steady state. We show that the species $U$ with heterogeneous distribution is always in a better position than its competitor $V$ with homogeneous distribution (Theorem 1.3). If the random diffusion rate of $\mathrm{V}$ is large (with $\mathrm{U}^{\prime} \mathrm{s}$ advection being weak), then species $\mathrm{U}$ prevails. Theorem 1.5 and Theorem 1.6 tell us that strong advection of $U$ may be of benefit to $V$. For any fixed random diffusion, the two competition species always coexist for strong advection of $\mathrm{U}$.

Recently, in their wonderful works [1], Averill et al. found out the critical values $1 / \min _{\bar{\Omega}} \mathrm{m}$ and $1 / \max _{\bar{\Omega}} \mathrm{m}$ for the ratio of advection and random diffusion (see Lemma 4.1 and Remark 4.3 in [1]). These values turned out to be crucial to determine the dynamics with intermediate advection (see, for example Theorems 2.2 and 2.4 in [1]). Using these new observations, the main results in this paper can be restated and refined by the term of the ratio of advection and diffusion. In the other hand, motivated by the series of works of He and $\mathrm{Ni}([14-17])$, the models suggested in this paper remain many questions to be studied. Part of the results in this direction are contained in our next paper.

\section{Acknowledgment}

We are very grateful to an anonymous reviewer for the carefully readings and helpful suggestions which help improve our manuscript.

\section{References}

[1] I. Averill, K.-Y. Lam, Y. Lou, The role of advection in a two-species competition model: a bifurcation approach, American Mathematical Society, New York, (2017). 1, 2, 4

[2] F. Belgacem, C. Cosner, The effects of dispersal along environmental gradients on the dynamics of populations in heterogeneous environment, Canad. Appl. Math. Quart., 3 (1995), 379-397. 1

[3] R. S. Cantrell, C. Cosner, Spatial Ecology via Reaction-Diffusion Equations, John Wiley and Sons, Chichester, (2003). $1,1,1,2.1$

[4] R. S. Cantrell, C. Cosner, Y. Lou, Movement towards better enviromentsand the evolution of rapid diffusion, Math. Biosciences, 204 (2006), 199-214. 1

[5] R. S. Cantrell, C. Cosner, Y. Lou, Advection mediated coexistence of competing species, Proc. Roy. Soc. Edinburgh Sect., 137 (2007), 497-518. 3

[6] X.-F. Chen, R. Hambrock, Y. Lou, Evolution of conditional dispersal: A reaction-diffusion-advection model, J. Math. Biol., 57 (2008), 361-386.

[7] X.-F. Chen, Y. Lou, Principal eigenvalue and eigenfunctions of an elliptic operator with large advection and its application to a competition model. Indiana Univ. Math. J., 57 (2008), 627-658.

[8] X.-F. Chen, Y. Lou, Effects of diffusion and advection on the smallest eigenvalue of an elliptic operator and their applications, Indiana Univ. Math. J., 61 (2012), 45-80.

[9] C. Cosner, Y. Lou, Does movement toward better environments always benefit a population, J. Math. Anal. Appl., 277 (2003), 489-503. 1

[10] J. Dockery, V. Hutson, K. Mischaikow, M. Pernarowski, The evolution of slow dispersal rates: a reaction-diffusion model, J. Math. Biol., 37 (1998), 61-83. 1, 1

[11] D. Gilbarg, N. Trudinger, Elliptic Partial Differential Equations of Second Order, Springer, Berlin, (1983). 3

[12] R. Hambrock, Y. Lou, The evolution of conditional dispersal strategy in spatially heterogeneous habitats, Bull. Math. Biol., 71 (2009), 1793-1817. 1 
[13] A. Hastings, Can spatial variation alone lead to selection for dispersal, Theor. Pop. Biol., 24 (1983), 244-251. 1, 1

[14] X. He, W.-M. Ni, The effects of diffusion and spatial variation in Lotka-Volterra competition-diffusion system, I: Heterogeneity vs. homogeneity, J. Differential Equations, 254 (2013), 528-546. 1, 1, 1, 1.2, 1, 4

[15] X. He, W.-M. Ni, The effects of diffusion and spatial variation in LotKa-Volterra competition-diffusion system, II: The general case, J. Differential Equations, 254 (2013), 4088-4108. 1, 1

[16] X. He, W.-M. Ni, Global dynamics of the Lotka-Volterra competition-diffusion system: Diffusion and spatial heterogeneity, I, Comm. Pure Appl. Math., 69 (2016), 981-1014.

[17] X. He, W.-M. Ni, Global dynamics of the Lotka-Volterra competition-diffusion system with equal amount of total resources, II, Calc. Var. Partial Differential Equations, 2016 (2016), 20 pages. 1, 1, 1, 1.2, 1, 1, 4

[18] P. Hess, Periodic-parabolic Boundary Value Problems and Positivity, Longman Scientific \& Technical, Harlow, (1991). 2.4

[19] M. W. Hirsch, Stability and convergence in strongly monotone dynamical systems, J. Reine Angew. Math., 383 (1988), 1-53. 3

[20] S. Hsu, H. Smith, P. Waltman, Competitive exclusion and coexistence for competitive systems on ordered Bnanch spaces, Trans. Amer. Math. Soc., 348 (1996), 4083-4094. 1

[21] Y.-L Huang, C.-H. Wu, Positive steady states of reaction-diffusion-advection competion models in periodic environment, J. Math. Anal. Appl., 453 (2017), 724-745. 4

[22] K.-Y. Lam, Concentration phenomena of a semilinear elliptic equation with large advection in an ecological model, J. Differential Equations, 250 (2011), 161-181. 1

[23] K.-Y. Lam, Limiting profiles of semilinear elliptic equations with large advection in population dynamics, II, SIAM J. Math. Anal., 44 (2012), 1808-1830.

[24] K.-Y. Lam, W.-M, Ni, Limiting profiles of semilinear elliptic equations with large advection in population dynamics, Discrete Contin. Dyn. Syst., 28 (2010), 1051-1067. 1

[25] K.-Y. Lam, W.-M, Ni, Uniqueness and complete dynamics in the heterogeneous competition-diffusion systems, SIAM J. Appl. Math., 72 (2012), 1695-1712. 2.3, 2.4, 3

[26] K.-Y. Lam, W.-M. Ni, Advection-mediated competition in general environments, J. Differential Equations, 257 (2014), 3466-3500. 2

[27] Y. Lou, On the effects of migration and spatial heterogeneity on single and multiple species, J. Differential Equations, 223 (2006), 400-426. 3

[28] Y. Lou, Some challenging mathematical problems in evolution of dispersal and population dynamics, Springer, Berlin, (2008). 1

[29] M. H. Protter, H. F. Weinberger, Mximum Principles in Differential Equations, Springer, Berlin, (1984). 1, 3

[30] H. Smith, Monotone Dynamical Systems, An Introduction to the Theory of Competitive and Cooperative Systems, American Mathematical Society, Providence, (1995). 2, 2.3 